\title{
Acute osteomyelitis of the rib in a neonate
}

\author{
Nalika de Silva ${ }^{1}$, Dhammika Sirisena Vidanagama ${ }^{2}$, Tolusha Harischandra ${ }^{3}$, Sujeewa Kariyawsam ${ }^{4}$, Charith \\ Rathuwithana $^{5}$
}

Sri Lanka Journal of Child Health, 2014; 43(2): 104-105

(Key words: Acute osteomyelitis; neonate; rib; MRSA)

\section{Case report}

A one month old baby girl presented to Base Hospital, Balapitiya with a 2 day history of moaning, fever and reduced activity. She was born at term with a birth weight of $2640 \mathrm{~g}$ and had an uneventful antenatal and perinatal period. She was thriving well until 2 days back. On examination, baby was febrile and irritable. Hydration was good with normal capillary refill time. Systemic examination, including movement of all joints, was normal.

Intravenous (IV) penicillin $\mathrm{G}$ and gentamicin were started after taking blood for culture. Full blood count (FBC) and urine full report were normal and Creactive protein (CRP) was $24 \mathrm{mg} / \mathrm{L}$. Cerebrospinal fluid (CSF) analysis was normal. Ultrasound scans (USS) of brain, abdomen and hip joints were normal. Following IV antibiotics, baby improved clinically.

On day four, the blood culture grew Staphylococcus aureus and antibiotics were changed to IV cloxacillin. The antibiotic sensitivity test, received subsequently, showed the organism to be MRSA sensitive to vancomycin, amikacin, cotrimoxazole and fusidic acid and IV amikacin was added. Markers of infection were repeated in view of MRSA culture. CRP was $84 \mathrm{mg} / \mathrm{L}$ and $\mathrm{FBC}$ and blood picture showed increased neutrophil leukocytosis with evidence of severe bacterial infection. An echocardiogram was done to rule out cardiac vegetations. On day 5 of IV

$\overline{{ }^{1} \text { Consultant Paediatrician, Base Hospital, Balapitiya, }}$, ${ }^{2}$ Consultant Microbiologist, Teaching Hospital, Karapitiya, ${ }^{3}$ Consultant Cardiothoracic Surgeon, Teaching Hospital, Karapitiya, ${ }^{4}$ Consultant Radiologist, Base Hospital Balapitiya, ${ }^{5}$ Senior House Officer in Paediatrics, Base Hospital Balapitiya

(Received 23 April 2013: Accepted after revision on 21 June 2013) amikacin, CRP was normal and FBC and blood picture were resolving satisfactorily. The same day the baby developed a lump over the left side of chest and chest x-ray (CXR) and USS revealed acute osteomyelitis of the left $5^{\text {th }}$ rib (Figure 1).

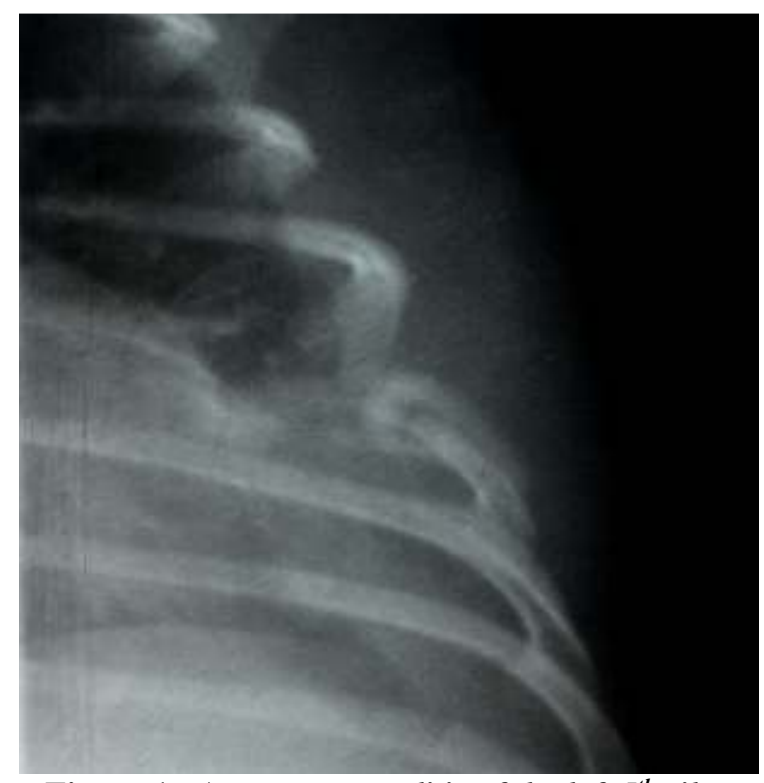

Figure 1: Acute osteomyelitis of the left $5^{\text {th }}$ rib

Baby was transferred to Teaching Hospital Karapitiya where computed tomography (CT) scan further confirmed the diagnosis. Left anterior axillary mini-thoracotomy was done. She was found to have a sequestrum which was removed with the periosteum left behind. Antibiotics were changed to IV vancomycin. Baby was retransferred and IV vancomycin was continued for 14 days. Bone culture and repeat blood cultures were sterile and histology confirmed acute osteomyelitis. On discharge baby had started to gain weight. Follow up visit showed a thriving baby with a normal CRP and a regrown $5^{\text {th }}$ rib (Figure 2). 


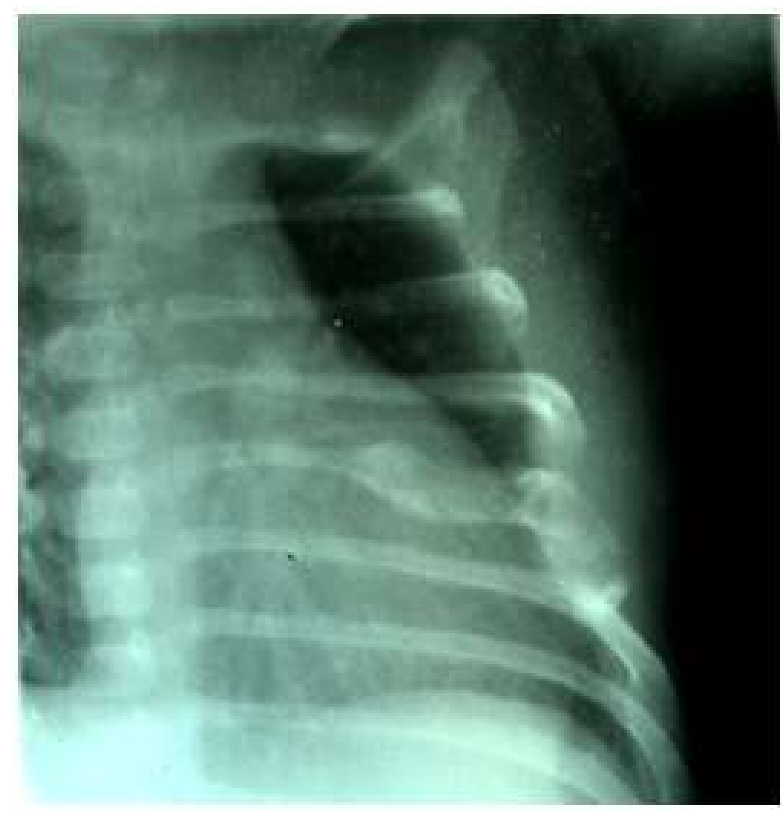

Figure 2: 3 weeks after surgical removal of part of $L / 5^{\text {th }}$ rib

\section{Discussion}

Neonatal osteomyelitis is uncommon, and most frequently involves the humerus and femur ${ }^{1}$. Rib osteomyelitis occurs in approximately $1 \%$ or less of all cases of haematogenous osteomyelitis ${ }^{2}$. Dissemination of neonatal osteomyelitis in the majority of cases is haematogenous. Extension from an adjacent focal infection, direct inoculation of bone following trauma/surgery or secondary to vascular insufficiency are other forms of infection ${ }^{1}$.

The most common organism isolated is Staph. aureus $s^{1,3}$. Infection caused by community acquired MRSA is becoming an increasingly common problem ${ }^{3}$. MRSA can spread via nosocomial, familial and mother to infant transmission.

Clinical presentation of neonatal acute osteomyelitis is usually non-specific and causes delay in diagnosis. There is a benign clinical onset in the majority of neonates with little or no evidence of infection ${ }^{4}$. Common clinical features include soft tissue swelling, tenderness and decreased motion. Systemic manifestations of sepsis are rarely present ${ }^{1}$.

Microbiology and imaging are equally important in confirming the diagnosis of acute osteomyelitis. A bacteriological diagnosis can be made in $50-80 \%$ of cases if blood and bone cultures are obtained ${ }^{3}$. Radiographic findings of osteomyelitis usually require 7 to 14 days to appear, and are readily confirmed by the presence of expansion and destruction of the rib, patchy necrosis, and extrapleural swelling of soft tissue. Occasionally a sequestrum may be present.

Radioisotope bone scanning, ultrasonography of bone, CT scan, and magnetic resonance imaging (MRI) scan are useful in making an early diagnosis. Successful treatment of osteomyelitis depends on the appropriate selection and administration of antibiotics and surgical intervention as needed. When culture results are available, antibiotic therapy should be modified according to the organism and the susceptibility pattern. Vancomycin administered intravenously is the gold standard for treating osteomyelitis due to MRSA infection ${ }^{5}$. Resection of the rib offers the best treatment for pyogenic rib osteomyelitis ${ }^{6}$.

\section{References}

1. Korakaki E, Aligizakis A, Manoura A, Hatzidaki E, Saitakis E, Anatoliotaki M, et al. Methicillinresistant staphylococcus aureus osteomyelitis and septic arthritis in neonates: Diagnosis and management. Japanese Journal of Infectious Disease 2007; 60 (2-3): 129-31.

2. Naik-Mathuria B, Ng G, Olutoye OO. Lytic rib lesion in a 1-year-old child: Group A beta streptococcal osteomyelitis mimicking tumor. Pediatric Surgery International 2006; 22(10):837-9. http://dx.doi.org/10.1007/s00383-006-1725-5

3. Gutierrez K. Bone and joint infections in children. Pediatric Clinics of North America 2005; 52: 779-94. http://dx.doi.org/10.1016/j.pcl.2005.02.005

4. Knudsen CJM, Hoffman EB. Neonatal osteomyelitis. The Journal of Bone and Joint Surgery 1990; 72B: 846-51.

5. Kaplan SL. Osteomyelitis in children Infectious Disease Clinics of North America 2005; 19: 787-97. http://dx.doi.org/10.1016/j.idc.2005.07.006

6. Osinowo O, Adebo OA, Okubanjo OA. Osteomyelitis of the ribs in Ibadan. Thorax 1986; 41: 58-60. http://dx.doi.org/10.1136/thx.41.1.58 